\title{
Modelo conceitual para jogos educativos digitais
}

\section{A conceptual model for digital educational games}

\author{
Rafael Feyh Jappur ${ }^{1}$, Fernando Antonio Forcellini ${ }^{2}$, Fernando Jose Spanhol ${ }^{2}$ \\ ${ }^{1}$ Faculdade SENAC Florianópolis, Florianópolis, SC, Brasil \\ 2 Universidade Federal de Santa Catarina (UFSC), Florianópolis, SC, Brasil
}

Autor para correspondência/Corresponding author: Rafael Feyh Jappur [ rjappur@gmail.com ]

Recebido/Submitted: 12 Nov. 2014

Aceito/Approved: 13 Dez. 2014

Copyright (c) 2014 Jappur, Forcellini \& Spanhol. Todo o conteúdo da Revista está sob uma licença Creative Commons Atribuição-NãoComercial-Compartilhalgual 3.0 Não Adaptada. Ao serem publicados por esta Revista, os artigos são de livre uso em ambientes educacionais, de pesquisa e não comerciais, com atribuição de autoria obrigatória. Mais informações em http://www.atoz.ufpr.br/index.php/atoz/about/submissions\#copyrightNotice.

\section{Resumo}

Introdução: Apresenta o desenvolvimento de uma proposta de modelo conceitual para jogos educativos digitais no contexto da sala de aula, tendo como foco de análise a aprendizagem para hábitos sustentáveis de consumo e produção no ambiente residencial. Os modelos conceituais existentes estão mais ligados à criação e recentemente à avaliação dos jogos. Já no contexto da aplicação, a maioria dos modelos foca primordialmente na descrição dos processos de validação dos jogos do que na mediação da prática pedagógica em si. Portanto, a questão de pesquisa versa sobre em como criar, aplicar e avaliar jogos educativos digitais para o processo de ensino e aprendizagem em sala de aula, tendo como foco de aprendizagem a cultura da sustentabilidade no ambiente residencial.

Método: Realizou-se pesquisa bibliográfica e de campo como parte prática para a coleta de dados. Ressalta-se o uso do Design Science Research Methodology como procedimento metodológico para o desenvolvimento do modelo conceitual.

Resultados: O mesmo foi testado em um estudo piloto em duas turmas do programa jovem aprendiz do SENAC/SC para verificação de sua consistência.

Conclusão: Conclui-se com base nos resultados apresentados pela aplicação do piloto que o modelo conceitual proposto contribui para o processo de ensino e aprendizagem em sala de aula.

Palavras-chave: Jogos educativos digitais. Modelo conceitual. Processos de ensino e aprendizagem. Sustentabilidade.

\begin{abstract}
Introduction: It presents the development of a proposed conceptual model for digital educational games in the context of the classroom in which the analysis focused on learning for sustainable habits production and consumption in the residential environment. The existing conceptual models are more linked to the creation and recently with evaluation of the games. In the context of the implementation, most models focus more on the description of the games validation processes than in mediating the pedagogical practice itself. Therefore, the research question is about how to create, implement and evaluate digital educational games for teaching and learning process in the classroom, with the focus on learning the culture of sustainability in the residential environment.

Method: As far as the methodological characterization is concerned, the investigation was supported by bibliographical and field research for data collection. It was emphasized the use of Design Science Research Methodology as methodological procedure for the development of the conceptual model. Results: The system has been tested in a pilot study in two groups of Young Apprentice Program at SENAC/SC to check its consistency.

Conclusion: It was concluded based on the results produced by the application of the pilot that the proposed conceptual model contributes to the process of teaching and learning in the classroom.
\end{abstract}

Keywords: Digital educational games.Conceptual model. Teaching and learning process. Sustainability.

\section{INTRODUÇÃO}

$\mathrm{O}$ artigo aborda o contexto do uso de jogos educativos digitais no processo de ensino e aprendizagem em sala de aula, tendo com foco a aprendizagem para a cultura da sustentabilidade, especificamente para o ambiente residencial, caracterizado pelos hábitos de consumo e produção humana em edificações. Sendo estas vinculadas, segundo Jappur, Forcellini e Selig (2010), a vários aspectos ambientais gerados em edificações, tais como: a produção de resíduos sólidos e líquidos, e o consumo de energia e água.

Os jogos educativos digitais podem ser um agregador para o ensino de conteúdos e para o processo de aprendizagem em sala de aula, ligados à cultura da sustentabilidade. Todavia, constata-se - conforme apresentam Kirriemuir e Mcfarlane (2004); Balasubramanian e Wilson, (2006); Baek (2008), Echeverría et al. (2011), entre outros - que os professores ou mediadores possuem dificuldades para aplicar os jogos em sala de aula.

Balasubramanian e Wilson (2006) apontam que os jogos educativos digitais ainda são pouco utilizados na escola e, para muitos educadores, o desafio é encontrar e utilizar bons jogos como ferramenta de aprendizagem. Para os autores, os jogos digitais, no seu processo de criação têm sido usados de forma não articulada aos princípios e necessidades pedagógicas. Isto muitas vezes gera fragilidades ou dificuldades no que tange à seleção e aplicação pelos educadores, por não compreenderem o valor agregado à aprendizagem (Balasubramanian \& Wilson, 2006). 
Constata-se, ainda, que outras dificuldades para a aplicação dos jogos educativos digitais no contexto da sala de aula estão ligadas a falta dos princípios pedagógicos na criação dos jogos, seja pela falta de mediação na aplicação, ou até mesmo da avaliação da eficiência e eficácia do uso dos jogos em sala de aula (Baek, 2008; Balasubramanian \& Wilson, 2006; Echeverría et al., 2011; Kirriemuir \& Mcfarlane, 2004).

O problema de modelar jogos educativos digitais é salientado por alguns autores, tais como Hsiao (2007), Moreno-Ger, Burgos, Martínez-Ortiz, Sierra e Fernández-Manjóna (2008), Aleven, Myers, Easterday e Ogan, (2010), Echeverría et al. (2011), Villalta et al. (2011) e Savi (2011). Porém, verifica-se na literatura consultada que existem lacunas de como criar, aplicar e avaliar os jogos educativos digitais para o contexto do processo de ensino e aprendizagem em sala de aula. Também se constata que os modelos pesquisados estão mais ligados à criação e recentemente com a avaliação dos jogos. Já no contexto da aplicação, a maioria dos modelos pesquisados focam na descrição dos processos de validação dos jogos, do que sobre a mediação da prática pedagógica em si (Hsiao, 2007; Savi, 2011).

O presente artigo, portanto, pretende apresentar uma proposta de modelo conceitual que estruture de forma integrada os processos para a criação, aplicação e avaliação de jogos educativos digitais, no contexto da sala de aula, tendo como foco de análise a aprendizagem para hábitos sustentáveis de consumo e produção no ambiente residencial.

Na sequência, apresenta-se a metodologia utilizada e uma breve descrição teórica sobre os jogos educativos digitais, para em seguida apresentar a proposta de modelo conceitual e a aplicação do teste piloto, assim como as considerações finais.

\section{JOGOS EDUCATIVOS DIGITAIS}

De acordo com Huizinga (2007), o jogo é uma atividade realizada dentro de um limite de espaço e tempo, segundo regras livremente consentidas, dotado de um fim em si mesmo, e acompanhado de um sentimento de tensão e alegria com consciência da diferença da vida cotidiana.

Criar um jogo que seja divertido e educativo (digital ou não digital) é um desafio significativo. No entanto, muito pouco tem sido escrito sobre como projetar jogos educativos eficazes (Aleven, Myers, Easterday, \& Ogan, 2010; Echeverría et al., 2012; Schell, 2008).

Os jogos digitais quando preparados para o contexto educacional podem receber diferentes nomenclaturas (Savi, 2011). Neste artigo se utilizará - preponderantemente - a denominação de jogos educativos digitais, ainda que não de forma limitada.

A produção científica sobre jogos educativos digitais vem crescendo nas últimas duas décadas com pesquisas sobre o potencial destes jogos para o processo de ensino e aprendizagem (Hsiao, 2007; Moreno-Ger et al., 2008; Villalta et al., 2011).

O uso destes jogos como ferramenta educacional está lentamente se tornando uma prática aceita em ambientes de aprendizagem. Eles apresentam diversas possibilidades para o desenvolvimento do conhecimento e também ajudam a melhorar o processo de ensino e aprendizagem nas escolas (Villalta et al., 2011).

Contudo, segundo Balasubramanian e Wilson (2006), os jogos educativos digitais ainda são pouco utilizados e para muitos educadores encontrar e utilizar bons jogos são um desafio. Isso ocorre, em boa parte, porque muitos dos jogos têm feito uso limitado dos princípios pedagógicos e acabam sendo ignorados pelos educadores por agregarem pouco valor às aulas.

Moreno-Ger et al. (2008) abordam que avaliação da aprendizagem dos alunos é outro impeditivo para o uso dos jogos educativos digitais pelos professores. Segundo os autores, é necessário verificar se os alunos estão atingindo os objetivos pedagógicos propostos e fornecer algum tipo de feedback, como por exemplo, relatórios informando ao aluno o seu desempenho, tempo, erros cometidos, entre outras informações. 
Além disso, várias questões como a relevância para currículo, precisão de conteúdos e compatibilidade da duração dos jogos com o horário de uso dos laboratórios de informática, têm impedido que os jogos educativos digitais se tornem uma atividade predominante nas instituições de ensino (Kirriemuir \& Mcfarlane, 2004).

Contudo, a proposta de modelo conceitual aqui apresentada foi desenvolvida, em especial, com base nos modelos de Klein, Nir-Gal, Darom (2000), Gomes (2001), Aleven et al. (2010), Echeverría et al. (2011), Villalta et al. (2011), Savi (2011), que se relacionam com a criação, aplicação e avaliação de jogos educativos digitais para o processo de ensino e aprendizagem em sala de aula (Quadro 1). Ressalta-se que os autores pesquisados não fazem uma distinção clara sobre a criação, aplicação e avaliação, como proposto neste modelo, porém, apresentam diretrizes a serem consideradas.

\section{Modelos de Jogos Educativos Digitais}

Com base na revisão bibliográfica e no estudo mais aprofundado dos modelos selecionados nesta pesquisa (Quadro 1) nos quais se constatou um consenso entre os autores - segundo os quais, para um bom jogo educativo digital é importante que haja uma proposta educacional e lúdica. Para Echeverría et al. (2011) e Villalta et al. (2011), a dimensão lúdica se refere aos elementos do jogo, respeitando, todavia, as questões impostas pela dimensão educativa ou pedagógica. Aleven et al. (2010) afirmam que um bom jogo educativo digital deve possuir um projeto pedagógico e um projeto de jogo divertido. Nesse sentido, elencaram-se as propostas pedagógicas e lúdicas, apresentadas pelos autores dos modelos identificados e selecionados nesta pesquisa (Quadro 1).

\begin{tabular}{|c|c|c|}
\hline Modelos Conceituais & Propostas Pedagógicas & Propostas Lúdicas \\
\hline Mayer, Moreno (2002) & $\begin{array}{l}\text { Taxionomia de Bloom Revisada (Anderson \& } \\
\text { Krathwohl, 2001). }\end{array}$ & Não apresenta. \\
\hline Echeverría et al. (2011) & $\begin{array}{l}\text { Taxionomia de Bloom revisada (Anderson \& } \\
\text { Krathwohl, 2001). }\end{array}$ & $\begin{array}{l}\text { Mecânica, história, estética, tecnologia (Schell, } \\
\text { 2008). }\end{array}$ \\
\hline Aleven et al. (2010) & $\begin{array}{l}\text { Taxionomia de Bloom revisada /Princípios do } \\
\text { design instrucional (Anderson \& Sosniak, 1995; } \\
\text { Mayer \& Moreno, 2003). }\end{array}$ & $\begin{array}{l}\text { Mecânica, dinâmica, estética (Hunicke, Leblanc, \& } \\
\text { Zubek, 2004). }\end{array}$ \\
\hline Villalta et al. (2011) & $\begin{array}{l}\text { Não apresentam proposta única, mas devem } \\
\text { estar alinhadas com as estratégias instrucionais. }\end{array}$ & $\begin{array}{l}\text { Apresenta um guia de avaliação, que inclui: } \\
\text { mecânica, progressão, metodologia, colaboração, } \\
\text { informação na tela, holismo. }\end{array}$ \\
\hline Savi (2011) & $\begin{array}{l}\text { Três primeiros níveis da taxionomia de Bloom } \\
\text { (conhecimento, compreensão e aplicação) } \\
\text { (Bloom, Engelhart, Furst, Hill, \& Krathwohl, } \\
\text { 1956); aprendizagem de curto e longo prazo } \\
\text { (Moody \& Sindre, 2003). }\end{array}$ & $\begin{array}{l}\text { Percepção dos alunos - Nível } 1 \text { Kirkpatrick, } \\
\text { 1994); Modelo A, R, C, S - Atenção, relevância, } \\
\text { competência e satisfação (Keller, 1987); Imersão, } \\
\text { interação social, desafio, divertimento e com- } \\
\text { petência (Gámez, 2009; Poels, Kort, \& ljsselsteijn, } \\
\text { 2007; Sweetser \& Wyeth, 2005; Takatalo, Häkkin- } \\
\text { en, Kaistinen, \& Nyman, 2010). }\end{array}$ \\
\hline Klein et al. (2000) & $\begin{array}{l}\text { Feuerstein - Seis critérios de mediação: inten- } \\
\text { cionalidade e reciprocidade, transcendência, } \\
\text { significado, competência e autorregulação } \\
\text { (Feuerstein, 1980; Klein, 1996). }\end{array}$ & Não apresenta. \\
\hline Campos e Macedo (2011) & $\begin{array}{l}\text { Feuerstein - Três critérios de mediação: inten- } \\
\text { cionalidade e reciprocidade, transcendência, } \\
\text { significado (Feuerstein, 1980). }\end{array}$ & Não apresenta. \\
\hline Gomes (2001) & $\begin{array}{l}\text { Mediação - todos os critérios de mediação (12 } \\
\text { critérios); Processo cognitivo; mecanismos de } \\
\text { aprendizagem (Feuerstein, 1980). }\end{array}$ & Não apresenta. \\
\hline
\end{tabular}

Quadro 1. Propostas pedagógicas e lúdicas.

Fonte: Elaboração própria.

No quadro 1 pode-se verificar, no que se refere às propostas pedagógicas, que os trabalhos de Mayer e Moreno (2002), Echeverría et al. (2011) e Aleven et al. (2010) utilizam a taxionomia revisada de Bloom (Anderson \& Krathwohl, 2001). Já Savi (2011) aplicou os três primeiros níveis da taxionomia de Bloom de 1954 na versão original (conhecimento, compreensão e aplicação) (Bloom, Engelhart, Furst, Hill, \& Krathwohl, 1977), e o modelo de aprendizagem de curto e longo prazo de Moody e Sindre (2003). Aleven et al. (2010) incorporaram, em seu estudo, os princípios do design instrucional (Anderson \& Sosniak, 1995; Mayer \& Moreno, 2002; Moreno-Ger, 2008) e os objetivos pedagógicos da taxionomia revisada de Bloom (Anderson \& Krathwohl, 2001). 
Villalta et al. (2011), assim como Aleven et al. (2010), também apresentam a necessidade de os jogos estarem alinhados com as estratégias instrucionais e sistematizam seis diretrizes para um projeto de jogo na modalidade Classroom Multiplayer Presential Game (CMPG). Contudo, os autores argumentam que outros métodos e diretrizes podem ser integrados, a exemplo da taxonomia revisada de Bloom (Anderson \& Krathwohl, 2001), como uma estrutura que ajuda na definição dos objetivos da aprendizagem. Os autores citam o modelo de Echeverría et al. (2011) como exemplo.

Por sua vez, Klein et al. (2000), Campos e Macedo (2011) e Gomes (2001) apresentam como proposta pedagógica os critérios da Experiência da Aprendizagem Mediada (EAM) de Feuerstein (1980), principalmente os três critérios fundamentais para uma EAM, a saber: intencionalidade e reciprocidade, transcendência, significado. Os autores salientam que os outros critérios da proposta de Feuerstein (1980) também são importantes, pois agregam e motivam a realização ou efetivação dos critérios fundamentais ou principais, de modo a enriquecer o processo de mediação da aprendizagem.

Gomes (2001) descreve doze critérios para a aprendizagem mediada, estando estes em conformidade com os critérios de mediação de Feuerstein (1980). Além dos critérios para EAM, Gomes (2001) também descreve um processo cognitivo com sete parâmetros de análise, adaptados do mapa cognitivo de Feuerstein (1980), e os mecanismos de aprendizagem, compostos por oito estratégias para as análises pedagógicas. Campos e Macedo (2011) demonstram um conjunto de indicadores para os três critérios principais da EAM, com foco na mediação de jogos em sala de aula.

No que tange às propostas lúdicas, constatou-se que os modelos de Mayer e Moreno (2002), Klein et al. (2000), Campos e Macedo (2011) e Gomes (2001) não apresentaram propostas lúdicas específicas, muito embora tenham citado a importância dos aspectos lúdicos para os jogos.

Já Echeverría et al. (2011), baseados nos estudos de Schell (2008), descrevem quatro elementos lúdicos para um jogo educativo digital: a mecânica (procedimentos, regras, os objetivos etc.); a história (roteiro de eventos); a estética (design gráfico, cores, música, efeitos sonoros etc.), e a tecnologia (dispositivos, displays, plataformas etc.).

Aleven et al. (2010) utilizaram os estudos de Hunicke, Leblanc, e Zubek (2004) para descrever os três componentes básicos para o desenvolvimento de jogos: mecânica (materiais, regras, objetivos, movimentos básicos e opções de controle para os jogadores); dinâmica (comportamentos que resultam da aplicação da mecânica do jogo); e estética (sensação, fantasia, narrativa, desafio, camaradagem, descoberta, expressão e submissão).

Villalta et al. (2011) propõem seis categorias com diretrizes para superar problemas apresentados por jogos, sendo estes: mecânica (interatividade e orientação, mecânica ligada aos objetivos da aprendizagem); progressão do jogo (narrativa, aumento gradual da dificuldade); metodologia (o professor é o mediador); colaboração (interações, mecânica vinculada à colaboração); informação na tela (distribuição espacial, elementos reconhecíveis, linguagem acessível e cuidados com excesso de informações); e holismo (guia de ação que inclui os aspectos educativos e lúdicos).

Savi (2011) não aprofunda a questão relacionada aos elementos de criação de jogos educativos digitais, mas destaca o design instrucional como um guia mais utilizado pelos desenvolvedores. Em seu trabalho, o autor relaciona alguns requisitos para avaliar a percepção dos alunos em relação à qualidade dos jogos. Para tal, o autor utiliza o nível 1 (reação) do modelo de Kirkpatrick (1994). Sendo que, para avaliar a motivação dos usuários ou alunos em jogar, é utilizado o modelo A, R, C, S (atenção, relevância, confiança e satisfação) de Keller (1987); e para avaliar a experiência do usuário com os jogos, são utilizados os seguintes parâmetros: imersão, interação social, desafio, divertimento e competência (Gámez, 2009; Ijsselsteijn, 2007; Poels \& Kort, 2007; Sweetser \& Wyeth, 2005; Takatalo, Häkkinen, Kaistinen, \& Nyman, 2010). 


\section{METODOLOGIA}

A natureza desta pesquisa, em acordo com Silva e Menezes (2005), possui mais similaridades com a pesquisa aplicada, pois objetiva gerar conhecimentos para aplicação prática dirigida à solução de problemas vivenciados no ambiente residencial.

Quanto à forma de abordagem, o presente trabalho representa uma pesquisa qualitativa, pois suas características principais coincidem com as recomendações feitas por vários autores como Silva e Menezes (2005), Vergara (2009) e Gil (2010).

Quanto aos objetivos, situa-se em três categorias: exploratória, descritiva e explicativa. Segundo Vergara (2009) e Gil (2010), os tipos de pesquisa não são mutuamente exclusivos, o que permite classificá-lo nestes três tipos.

Os procedimentos metodológicos utilizados foram preponderantemente a pesquisa bibliográfica e de campo, com a utilização do Design Science Research Methodology (DSRM) para o desenvolvimento do modelo conceitual.

A Metodologia de Pesquisa da Ciência do Design (Design Science Research Methodology - DSRM) busca preencher a falta de uma metodologia para servir como modelo aceito e válido para o desenvolvimento de artefatos para a ciência da informação. A DSRM incorpora princípios, práticas, e procedimentos necessários para realizar tais pesquisas e apresenta seis etapas/atividades que seguem uma sequência nominal ou procedural (Peffers, Tuunanen, Rothenberger, \& Chatterjee, 2007):
a) identificação do problema e motivação;
b) definição dos objetivos;
c) design e desenvolvimento;
d) demonstração;
e) avaliação; e
f) comunicação.

Embora as atividades sejam apresentadas de forma sequencial, não impõem uma ordem exata para o início da pesquisa. A metodologia apresenta como principal diferencial o fato de ter sido concebida de modo a possibilitar que o início da pesquisa possa ocorrer em diferentes etapas, considerando o foco que se pretenda dar à investigação. Segundo Peffers et al. (2007) existem quatro pontos distintos para o início de uma pesquisa, Sendo estes: início gerado por problema; início gerado por solução; início gerado por projeto/design e desenvolvimento; e início gerado por cliente/contexto. Todavia, se a pesquisa iniciar de forma não sequencial, as atividades anteriores não desenvolvidas devem ser identificadas e alinhadas com o tema da pesquisa (Peffers et al., 2007).

O início desta pesquisa, conforme orienta o DSRM, foi o projeto e desenvolvimento do jogo educativo digital denominado de Simulador Ambiental (SA). A partir da experiência realizada com a criação do jogo foi concebido o modelo conceitual pretendido. O modelo conceitual foi testado em um estudo piloto em duas turmas do programa jovem aprendiz do SENAC/SC para verificação de sua consistência.

\section{PROPOSTA DE MODELO CONCEITUAL PARA JOGOS EDUCATIVOS DIGITAIS}

A proposta de modelo conceitual visa estabelecer uma estrutura de processos para a criação, aplicação e avaliação de jogos educativos digitais para o processo de ensino e aprendizagem em sala de aula (Figura 1).

O processo de criação envolve uma proposta pedagógica e lúdica para que os desenvolvedores criem bons jogos educativos digitais. A estrutura pedagógica propõe a utilização da taxionomia Revisada de Bloom conforme descrevem Anderson, Krathwohl (2001), Krathwohl (2002), Aleven et al. (2010), Villalta et al. (2011) e Echeverría et al. (2011) para a definição dos objetivos educacionais do jogo e de um guia de recomendações com os critérios de mediação de Feuerstein (1980), em conformidade com The International Center for the 
Enhancement of Learning Potential [ICELP] (2012) e Gomes (2001), adaptados para o contexto de criação dos jogos educativos digitais.

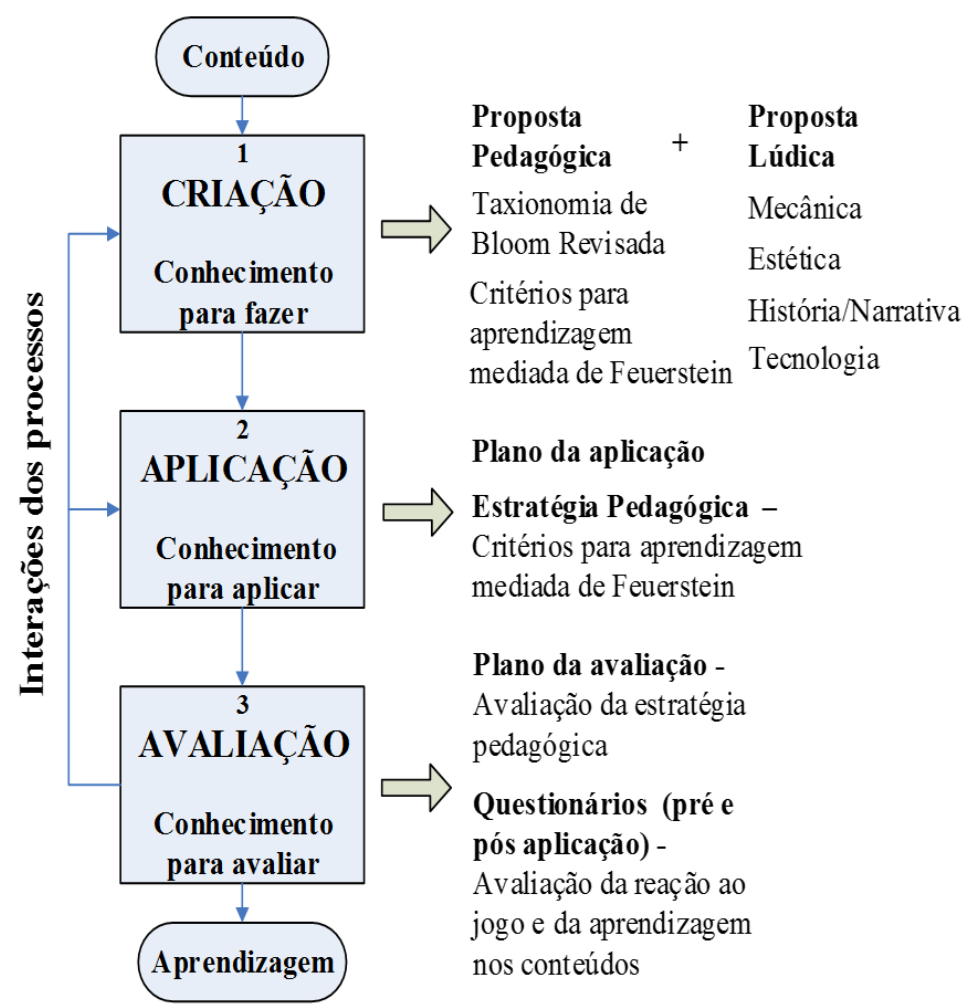

Figura 1. Modelo conceitual para jogos educativos digitais. Fonte: Elaboração própria.

Trata-se de uma proposta que visa incrementar o conhecimento didático-pedagógico dos desenvolvedores, com a intenção de apoiar os processos do design instrucional do jogo, para que criem uma mecânica de jogo favorável à mediação da aprendizagem do jogador em relação a um conteúdo específico.

Já a proposta lúdica para a criação dos jogos educativos digitais busca estabelecer um guia de recomendações com os elementos lúdicos a serem incorporados nos jogos. O guia lúdico é composto por quatro elementos principais, que são: mecânica; estética; história/narrativa; e tecnologia. A seleção destes elementos se basea nos trabalhos de Hunicke et al. 2004, Schell (2008), Aleven et al. (2010), Echeverría et al. (2011), Villalta et al. (2011).

O processo de aplicação do modelo conceitual estabelece uma estrutura para a mediação do processo de ensino e aprendizagem de conteúdos. Para tanto, baseado em Schön (2000), é utilizado um plano para a aplicação do jogo, que apresenta o conhecimento do mediador na ação pedagógica, a reflexão para a ação pedagógica e a reflexão na ação pedagógica. As estratégias pedagógicas devem ser descritas em conformidade com critérios da aprendizagem mediada de Feuerstein (1980), em acordo com Klein, Nir-Gal e Darom (2000), Gomes (2001, 2002), Mentis (2011), Souza, Machado, e Depresbiteris (2004), Campos e Macedo (2011) e ICELP (2012); sendo estes os mesmos apresentados para a criação, porém voltados para mediação do aprendizado do conteúdo do jogo durante a prática pedagógica.

O processo de avaliação do modelo conceitual esta estruturado por um plano de avaliação e por dois tipos de avaliações. Descrições e narrações, por meio da observação participante, para avaliar as estratégias pedagógicas (critérios de mediação de Feuerstein) utilizadas na prática pedagógica e a aplicação de três questionários para dar suporte para a coleta de dados para avaliação das variáveis reação e aprendizagem. O primeiro questionário é aplicado antes do jogo (pré-teste) para capturar a percepção dos jogadores a respeito de seu conhecimento no conteúdo do jogo; já o segundo e o terceiro deverão ser aplicados após o uso do jogo (póstestes). O segundo questionário visa conhecer a reação do jogador sobre a sua experiência e motivação com o jogo e se os objetivos educacionais apregoados para o mesmo foram atingidos; e o terceiro questionário é para verificar, em comparação com o primeiro, se o jogo possibilitou melhoria do aprendizado no conteúdo do jogo. 
O quadro 2 apresenta o referencial teórico do modelo conceitual que fundamenta o processo de avaliação dos jogos educativos digitais e das estratégias pedagógicas a serem utilizadas na prática pedagógica.

\begin{tabular}{|l|l|}
\hline Características & Referenciais teóricos \\
\hline Avaliaçães baseadas na percepção dos alunos. & Nível 1 e 2 do modelo de avaliação de treinamentos de Kirkpatrick (1994). \\
\hline Avaliação do nível de motivação dos jogos. & Modelo motivacional ARCS, desenvolvido por Keller (1987) e Savi (2011). \\
\hline Avaliação da experiência do usuário com os jogos. & $\begin{array}{l}\text { Modelos para avaliação da experiência do usuário em jogos (Gámez, 2009; Poels, } \\
\text { Kort, \& ljsselsteijn, 2007; Savi, 2011, Sweetser \& Wyeth, 2005; Takatalo et al., 2010). }\end{array}$ \\
\hline Avaliação dos objetivos educacionais dos jogos. & $\begin{array}{l}\text { Taxionomia revisada de Bloom (Anderson, Krathwohl, 2001; Bloom, 1956; Churches, } \\
\text { 2009; Echeverría et al., 2011; Krathwohl, 2002). }\end{array}$ \\
\hline $\begin{array}{l}\text { Avaliação da aprendizagem mediada com a prática } \\
\text { pedagógica. }\end{array}$ & $\begin{array}{l}\text { Critérios para a aprendizagem mediada de Feuerstein (Campos \& Macedo, 2011; } \\
\text { Feuerstein, 1980; Gomes, 2001, 2002; Klein et al., 2000; Mentis, 2011; Souza et al., } \\
\text { 2004; ICELP, 2012). }\end{array}$ \\
\hline
\end{tabular}

Quadro 2. Referenciais teóricos do processo de avaliação do modelo conceitual.

Fonte: Elaboração própria.

O quadro 3 apresenta a estrutura de avaliação do modelo conceitual. Sendo esta dividida em duas variáveis, cinco construtos e respectivas dimensões. Salienta-se que as dimensões do construto aprendizagem podem variar em função do conteúdo, temas e dos critérios da aprendizagem mediada utilizados na prática pedagógica.

\begin{tabular}{|c|c|c|}
\hline Variáveis & Construtos & Dimensões \\
\hline \multirow[t]{16}{*}{ Reação } & \multirow[t]{4}{*}{ Motivação } & Atenção \\
\hline & & Relevância \\
\hline & & Confiança \\
\hline & & Satisfação \\
\hline & \multirow[t]{6}{*}{ Experiência do usuário } & Imersão \\
\hline & & Desafio \\
\hline & & Competência \\
\hline & & Divertimento \\
\hline & & Controle \\
\hline & & Interação social \\
\hline & \multirow[t]{6}{*}{ Objetivos educacionais do jogo } & Lembrar \\
\hline & & Entender \\
\hline & & Aplicar \\
\hline & & Analisar \\
\hline & & Avaliar \\
\hline & & Criar \\
\hline \multirow[t]{5}{*}{ Aprendizagem } & $\begin{array}{l}\text { Conhecimentos no conteúdo (pré e pós- } \\
\text { teste) }\end{array}$ & Depende do conteúdo e dos temas apresentados pelo jogo \\
\hline & \multirow{4}{*}{$\begin{array}{l}\text { Estratégia pedagógica (Critérios para a } \\
\text { aprendizagem mediada de Feuerstein) }\end{array}$} & Intencionalidade e reciprocidade \\
\hline & & Significado \\
\hline & & Transcendência \\
\hline & & $\begin{array}{l}\text { Outros critérios da aprendizagem mediada de Feuerstein utilizados e } \\
\text { observados na prática pedagógica (depende do uso dos outros critérios) }\end{array}$ \\
\hline
\end{tabular}

Quadro 3. Estrutura do modelo conceitual para o processo de avaliação.

Fonte: Elaboração própria.

Portanto, o quadro 3 apresenta o resumo da estrutura do modelo conceitual para a avaliação da prática pedagógica e do jogo educativo digital utilizado. 
TESTE DA PROPOSTA DE MODELO CONCEITUAL PARA JOGOS EDUCATIVOS DIGITAIS

O teste piloto foi conduzido seguindo as seguintes etapas:

a) descrição do plano da aplicação e avaliação;

b) preparação dos materiais; apresentação do jogo e explicação dos questionários;

c) aplicação do questionário (pré-teste);

d) execução do jogo;

e) aplicação dos questionários (pós-testes); e

f) análise dos dados.

O teste piloto foi realizado na Faculdade SENAC Florianópolis, em parceria com o Projeto Recicle Ideias do SENAC Santa Catarina. A aplicação foi operacionalizada no laboratório de informática da Faculdade com duas turmas do Projeto Jovem Aprendiz (22 alunos - turma 804 e 16 alunos - turma 160), em períodos distintos. A média de idade dos alunos era entre 14 a 16 anos (Figura 2).

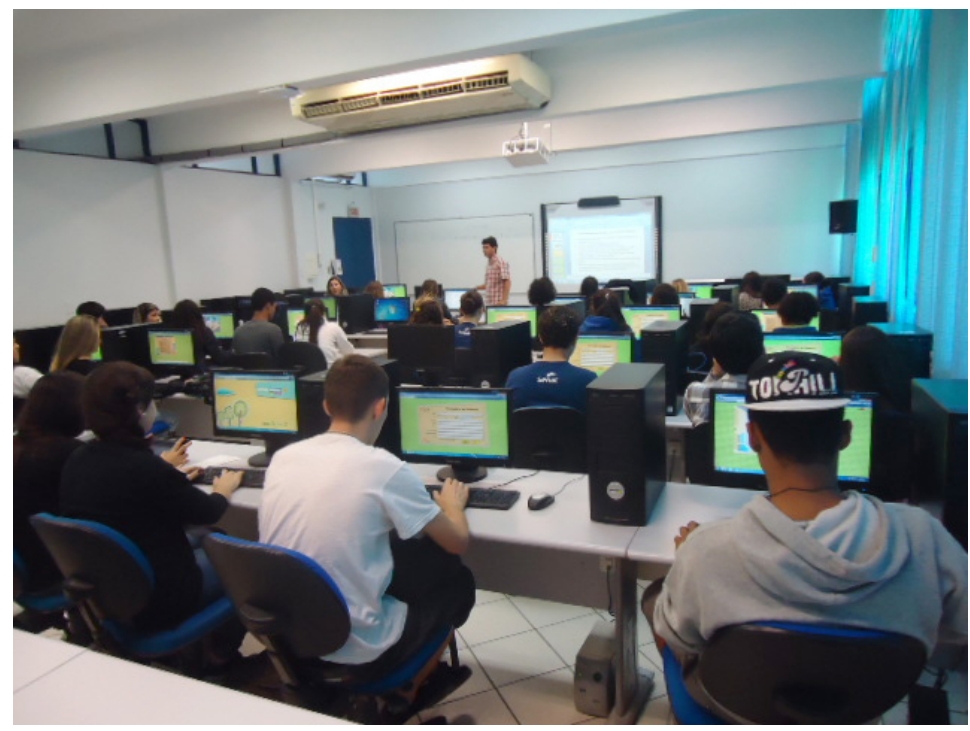

Figura 2. Aplicação turma 804.

Fonte: Elaboração própria.

O jogo educativo digital utilizado nas duas práticas pedagógicas foi o Simulador Ambiental (SA) (acesso http://www.meensina.org.br/site/simulador/) (Figura 3). Este jogo foi concebido junto ao desenvolvimento da proposta de modelo conceitual. O jogo visa à educação das pessoas para hábitos de consumo e produção mais sustentáveis no ambiente residencial. O contexto do SA está relacionado com a identificação e minimização dos impactos ambientais promovidos pelas ações humanas no ambiente residencial (Arbex, Jappur, Selig, \& Varvakis, 2012).

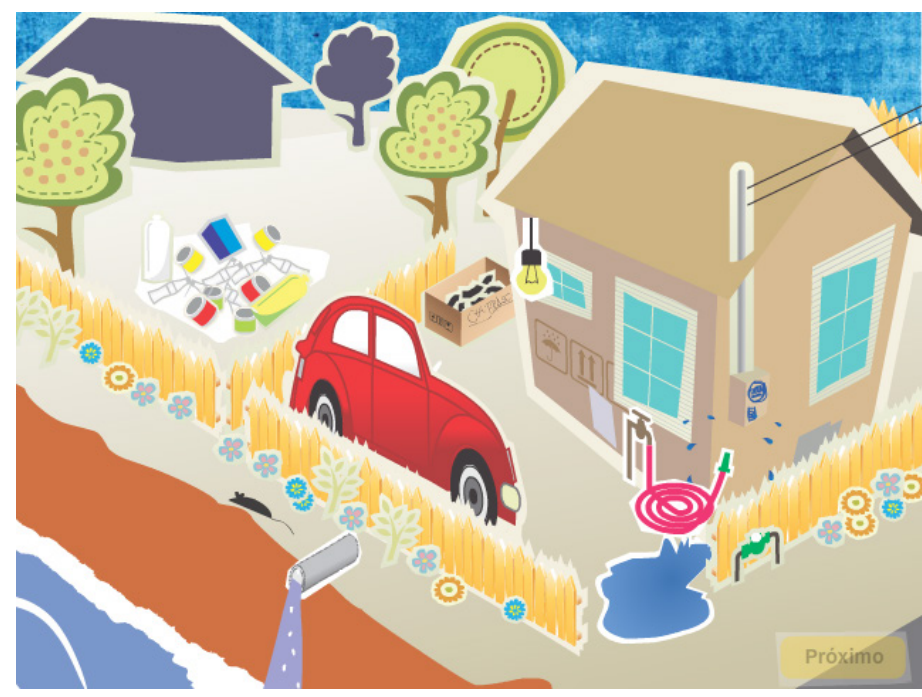

Figura 3. Ambiente da área externa da residência.

Fonte: Me Ensina, (2013). 
A proposta de jogo foi desenvolver alternativas para que o usuário pudesse navegar entre as telas dos ambientes de uma casa, facilitando a tomada de decisão em cada ambiente. O público alvo para qual o jogo foi projetado e desenvolvido é para o infanto-juvenil, não impossibilitando a utilização do jogo para outros públicos (Arbex et al., 2012).

Os itens dos questionários foram estabelecidos em acordo com o conteúdo do SA. O primeiro (pré-teste) e o terceiro (pós-teste) questionário foram elaborados com quatro questões para avaliar aprendizado dos jogadores no conteúdo. Já para o segundo questionário (pós-teste) elaboraram-se vinte questões para avaliar a reação dos alunos em relação à motivação, a experiência que o jogo proporcionou e se os objetivos pedagógicos foram alcançados.

Em referência aos resultados do aprendizado dos jogadores no conteúdo, constatou-se que houve melhoria no aprendizado dos alunos. Na primeira questão o aumento foi de aproximadamente em $13 \%$ e $27 \%$ nas turmas dos jovens aprendizes (804 e 106). Na segunda questão o aumento foi de aproximadamente em 12\% e 18\% nas turmas dos jovens aprendizes (804 e 106). A terceira questão apresentou aumento em $5 \%$ e igualmente em $5 \%$ nas turmas dos jovens aprendizes (804 e 106). A quarta questão apresentou aumento em $31 \%$ e $9 \%$ nas turmas dos jovens aprendizes (804 e 106).

O construto motivação, em praticamente todas as questões, obteve percentual acima de $70 \%$ de concordância, demonstrando que jogo teve um efeito positivo na motivação dos jogadores.

Os resultados do construto experiência do usuário no jogo proporcionaram reações positiva aos alunos, com destaque para as dimensões imersão, divertimento, competência e controle. Todavia, as dimensões interação social e desafio não foram tão bem avaliadas, demonstrando possibilidades de melhorias do jogo nestas dimensões. A dimensão interação social não foi tão bem avaliada, pois o jogo é individual, mesmo que os alunos compartilhem ideias durante o jogo. Já a dimensão desafio não teve boa pontuação, talvez pela linguagem e descrição das opções de escolha das questões arguidas nos ambientes do jogo. Porém, verifica-se uma possível contradição, pois 50\% dos alunos da turma 804, e 59\% dos alunos da turma 106 responderam que não concordam que obtiveram desempenho ótimo.

Os resultados apresentados dos objetivos educacionais atribuídos para o SA, segundo a percepção de mais de $70 \%$ dos alunos, demonstram que os mesmos concordam que o jogo ajuda a lembrar, entender, aplicar, analisar, avaliar e a criar conhecimento relacionado ao conteúdo do SA.

A estratégia pedagógica planejada para a aplicação do SA foi positiva, todos os critérios para a aprendizagem mediada foram trabalhados, conforme segue:

a) Intencionalidade e reciprocidade - As duas práticas pedagógicas sensibilizaram os participantes da importância dos hábitos sustentáveis de consumo e produção no ambiente residencial. Alguns problemas verificados como a gestão do tempo, velocidade da internet e o entendimento dos participantes quanto aos passos a serem seguidos não interferiram na reciprocidade das pessoas;

b) Significado - os participantes demonstraram terem entendido à razão da atividade de aprendizagem e a importância das tarefas. O planejamento das atividades valorizou as atitudes e as habilidades dos alunos, tais como: cooperação, argumentação, disciplina, respeito aos colegas, etc; e

c) Transcendência - A prática pedagógica com o uso do SA possibilitou aos participantes a fazerem analogias e debates de seus hábitos, desencadeando intenções para atitudes mais sustentáveis em suas residências.

Ressalta-se que os demais critérios da aprendizagem mediada também aconteceram, mesmo que de forma não planejada, foram utilizados em algum momento nas duas práticas pedagógicas. 


\section{CONCLUSÕES}

O estudo piloto apresentou dados preliminares positivos para a adequabilidade da sistemática do modelo conceitual. O envolvimento dos alunos na prática pedagógica, com o uso do SA, permitiu que eles efetivamente aprendessem hábitos sustentáveis de consumo e produção para suas residências. Todavia, estudos futuros devem ser empreendidos para aprofundamento do mesmo.

Contudo, constataram-se - durante a realização do piloto - algumas limitações referentes à gestão do tempo dos jogadores e dos recursos tecnológicos. O cronograma da execução da prática não foi totalmente conforme, pois alguns participantes levaram muito tempo para realizar o cadastro, devido à lentidão dos sistemas (cadastro dos participantes e envio dos logins e senhas para os e-mails pessoais), ou por terem esquecido a senha do e-mail pessoal. Outros levaram mais de dez minutos para responder aos questionários, por dificuldades no entendimento de como proceder.

Todavia, o presente trabalho oferece uma variedade de temas a serem aprofundados; no entanto, sugere-se para pesquisas futuras a aplicação do modelo conceitual em outros contextos, com temáticas e conteúdos diferentes da trabalhada neste artigo. Como por exemplo, jogos para aprendizagem em geografia, matemática, português, entre outros.

Durante a pesquisa bibliográfica várias abordagens pedagógicas foram identificadas, sendo sugeridos para trabalhos futuros outros estilos e teorias da aprendizagem. Referente a proposta lúdica, sugere-se aprimorar suas características, como por exemplo, o elemento estético do processo de criação de jogos, em relação à persuasão que determinados tipos de cores e sons provocam nos jogadores, motivando-os a jogar ou a ficar atentos a uma determinada situação.

Considera-se que há um amplo leque de possibilidades de novas aprendizagens mediadas por jogos educativos digitais, mas, destaca-se também que a figura central do professor-mediador deve ser incluída nesta nova forma didática de ensino e aprendizagem. 


\section{REFERÊNCIAS}

Aleven, V., Myers, E., Easterday, M., \& Ogan, A. (2010). Toward a framework for the analysis and design of educational games. IEEE International Conference on Digital Game and Intelligent Toy Enhanced Learning, 69-76. 2010. Retirado de http://delta. northwestern.edu/wordpress/wp-content/uploads/2013/11/ Aleven-2010-Toward-a-framework-for-the-analysis-anddesign-of-educational-games.pdf

Anderson, L. W., \& Krathwohl, D. R. (Eds.). (2001). A taxonomy for learning, teaching, and assessing: A revision of Bloom's taxonomy of educational objectives. New York: Longman.

Anderson, L. W., \& Sosniak, L. A. (Eds.). (1995). Bloom's taxonomy: A forty year retrospective. Chicago: University of Chicago.

Arbex, D., Jappur, R., Selig, P., \& Varvakis, G. (2012). Ergonomic aspects simulation digital online: An educational game proposal to promote environmental education. Work: a journal of prevention, assessment and rehabilitation, 41, 6011- 6015. doi:10.3233/WOR-2012-1052-6011

Baek, Y. (2008). What hinders teachers in using computer and video games in the classroom? Exploring factors inhibiting the uptake of computer and video games. Cyberpsychology \& Behavior, 11(6), 665-671 doi:10.1089/cpb.2008.0127

Balasubramanian, N., \& Wilson, B. G. (2006). Games and simulations. Society for Information Technology and Teacher Education International Conference. Retirado de http://site.aace.org/pubs/ foresite/GamesAndSimulations1.pdf

Bloom, B. S., Engelhart, M. D., Furst, E. J., Hill, W. H., \& Krathwohl, D. R. (Eds.). (1956). Taxonomy of educational objectives (V. 1). New York: David Mckay.

Bloom, B. S., Engelhart M. D., Furst, E. J., Hill, W. H., \& Krathwohl, D. R. (1977). Taxionomia de objetivos educacionais: Domínio cognitivo (6. Ed.). Porto Alegre: Globo.

Campos, M. C. R. M., \& Macedo, L. de. (2011). Desenvolvimento da função mediadora do professor em oficinas de jogos. Psicologia Escolar e Educacional, 15(2). doi:10.1590/S141385572011000200003

Churches, A. (2009). Bloom's digital taxonomy: Educational origami. Retirado de http://edorigami.wikispaces.com/ Bloom\%27s+and+ICT+tools

Echeverría, A., García-Campo, C., Nussbaum, M., Gil, F., Villalta, M., Améstica, M., \& Echeverría, S. (2011). A framework for the design and integration of collaborative classroom games. Computers \& Education, 57(1), 1127-1136. doi:10.1016/j. compedu.2010.12.010

Feuerstein, R. (1980). Instrumental enrichment: An intervention program for cognitive modifiability. Glenview: Scott, Foresman and Company.

Gámez, E. H. C. (2009). On the core elements of the experience of playing video games: Studying the gaming experience. LAP Lambert Academic Publishing, 2009.

Gil, A. (2010). Como elaborar projetos de pesquisa (5. Ed.). São Paulo: Atlas.

Gomes, C. M. A. (2001). Em busca de um modelo psico-educativo para $a$ avaliação de softwares educacionais. Dissertação de Mestrado, Universidade Federal de Santa Catarina, Brasil. Retirado de http://repositorio.ufsc.br/xmlui/handle/123456789/79700

Gomes. C. M. A. (2002). Feuerstein e a construção mediada do conhecimento. Porto Alegre: Artmed.

Hsiao, H. C. (2007). A brief review of digital games and learning. IEEE International Workshop on Digital Game and Intelligent Toy Enhanced Learning, 124-129. doi:10.1109/DIGITEL.2007.3
Huizinga, J. (2007). Homo ludens: O jogo como elemento da cultura. São Paulo: Perspectiva.

Hunicke, R., Leblanc, M., \& Zubek, R. (2004). MDA: A formal approach to game design and game research. AAAl Workshop Technical Report WS-04-04, 1-5. Retirado de http://www.cs.northwestern. edu/ hunicke/MDA.pdf

Jappur, R. F., Forcellini, F. A., \& Selig, P. M. (2010). Indicadores de gestão do conhecimento para sustentabilidade em edificações. 9. Congresso Brasileiro de Gestão do Conhecimento.

Keller, J. M. (1987). Development and use of the ARCS model of motivational design. Journal of Instructional Development, 10(3), 2-10.

Kirkpatrick, D. L. (1994). Evaluating training programs: The four levels. Berrett-Koehler Publishers.

Kirriemuir, J., \& Mcfarlane, A. (2004). Literature review in games and learning. Bristol: Futurelab. Retirado de http://www.mendeley. com/research/literature-review-in-games-and-learning/

Klein, S. P., Nir-Gal, O., \& Darom, E. (2000). The use of computers in kindergarten, with or without adult mediation; effects on children's cognitive performance and behavior. Computers in Human Behavior, 16(6), 591-608. doi:10.1016/S07475632(00)00027-3

Krathwohl, D. R. (2002). A revision of bloom's taxonomy: An overview. Theory into Practice, 41(4), 212-264. Retirado de http://www.celt.iastate.edu/teaching/RevisedBlooms1.html

Mayer, R. E., \& Moreno, R. (2002). Aids to computer-based multimedia learning. Learning and Instruction, 12(1), 107-119. doi:10.1016/ S0959-4752(01)00018-4

Mayer, R. E., \& Moreno, R. (2003). Nine ways to reduce cognitive load in multimedia. Learning Educational Psychologist, 38(1). doi:10.1207/S15326985EP3801_6

Me Ensina. (2013). Simulador ambiental. Retirado de http://www. meensina.org.br/site/simulador/

Mentis, M. (2011). Aprendizagem mediada dentro e fora de sala de aula. São Paulo: SENAC.

Moody, D., \& Sindre, G. (2003). Evaluating the effectiveness of learning interventions: an information systems case study. Proceedings of ECIS, Paper 80. Retirado de http://aisel.aisnet.org/ ecis $2003 / 80$

Moreno-Ger, P., Burgos, D., Martínez-Ortiz, I., Sierra, J. L., \& Fernández-Manjóna, B. (2008). Educational game design for online education. Computers in Human Behavior, 24(6), 25302540. doi:10.1016/j.chb.2008.03.012

Peffers, K., Tuunanen, T., Rothenberger, M. A., \& Chatterjee, S. (2007). A design science research methodology for information systems research. Journal of Management Information Systems, 24(3), 4577. doi:10.2753/MIS0742-1222240302

Poels, K., Kort, Y. D., \& ljsselsteijn, W. (2007). "It is always a lot of fun!": exploring dimensions of digital game experience using focus group methodology. ACM Conference on Future Play, 83-89.

Savi, R. (2011). Avaliação de jogos voltados para a disseminação do conhecimento. Tese de Doutorado, Universidade Federal de Santa Catarina, Brasil. Retirado de http://www.gqs.ufsc.br/wpcontent/uploads/2011/11/RafaelSavi_teseFinal_A5.pdf

Schell, J. (2008). The art of game design: A book of lenses. San Francisco: Morgan Kaufmann.

Schön, D. A. (2000). Educando o professional reflexivo: um novo design para o ensino e a aprendizagem. Artmed: Porto Alegre. 
Silva E. L., \& Meneses, E. M. (2005). Metodologia da pesquisa e elaboração de dissertação (4. Ed.). Florianópolis: PPGEP/UFSC.

Souza, A. M. M., \& Machado, O. T. M., \& Depresbiteris, L. (2004). A mediação como princípio deducional: Bases teóricas das abordagens de Reuven Feuerstein. São Paulo: Senac.

Sweetser, P., \& Wyeth, P. (2005). GameFlow: a model for evaluating player enjoyment in games. Computer Entertainment, 3(3), 1-24.

Takatalo, J., Häkkinen, J., Kaistinen, J., \& Nyman, G. (2010). Presence, involvement, and flow in digital games. In R. Bernhaupt (Ed.). Evaluating user experience in games: Concepts and methods. London: Springer.
The International Center for the Enhancement of Learning Potential (ICELP). (2012). Research: Basic theory. Retirado de http://www. icelp.org/asp/Basic_Theory.shtm

Vergara, S. C. (2008). Projetos e relatórios de pesquisa em administração (13. Ed.) São Paulo: Atlas.

Villalta. M., Gajardo, I., Nussbaum. M., Andreu, J. J., Echeverría, A., \& Plass, J. L. (2011). Design guidelines for classroom multiplayer presential games (CMPG). Computers \& Education, 57(3), 20392053. doi:10.1016/j.compedu.2011.05.003
Como citar este artigo (ABNT):

JAPPUR, R. F.; FORCELLINI, F. A.; SPANHOL, F. J. Modelo conceitual para jogos educativos digitais. AtoZ: novas práticas em informação e conhecimento, Curitiba, v. 3, n. 2, p. 116-127, jul./dez. 2014. Disponível em: <http://www.atoz.ufpr.br>. Acesso em:
How to cite this article (APA):

Jappur, R. R., Forcellini, F. A., \& Spanhol, F. J. (2014). Modelo conceitual para jogos educativos digitais. AtoZ: novas práticas em informação e conhecimento, 3(2), 116-127. Retrieved from http:// www.atoz.ufpr.br 